\title{
Stress-sensitive inference of task controllability
}

Authors: Romain Ligneul ${ }^{1,2 *}$, Zachary Mainen ${ }^{1}$, Verena $\mathrm{Ly}^{2,4 \dagger}$, Roshan Cools $^{2,3^{*} \dagger}$

\section{$\underline{\text { Affiliations: }}$}

${ }^{1}$ Champalimaud Research, Champalimaud Centre for the Unknown, Lisbon, Portugal.

${ }^{2}$ Radboud University, Donders Institute for Brain, Cognition and Behaviour, Centre for Cognitive Neuroimaging, Nijmegen, Netherlands.

${ }^{3}$ Radboud University Medical Centre, Department of Psychiatry, Nijmegen, Netherlands.

${ }^{4}$ Department of Clinical Psychology; Leiden Institute for Brain and Cognition, Leiden University, Leiden, The Netherlands.

* Correspondence to:

romain.ligneul@research.fchampalimaud.org

roshan.cools@fcdonders.ru.nl

$\dagger^{\dagger}$ Equal contribution. 


\begin{abstract}
Estimating environmental controllability enables agents to better predict upcoming events and decide when to engage controlled action selection. How does the human brain estimate environmental controllability? Trial-by-trial analysis of choices, decision times and neural activity in an explore-and-predict task demonstrate that humans solve this problem by comparing the predictions of an "actor" model with those of a reduced "spectator" model of their environment. Neural BOLD responses within striatal and medial prefrontal areas tracked the instantaneous difference in the prediction errors generated by these two statistical learning models. BOLD activity in the posterior cingulate, parietal and prefrontal cortices covaried with changes in estimated controllability. Exposure to inescapable stressors biased controllability estimates downward and increased reliance on the spectator model in an anxiety-dependent fashion. Taken together, these findings provide a mechanistic account of controllability inference and its distortion by stress exposure.
\end{abstract}




\section{INTRODUCTION}

Influential theories suggest that the human brain navigates its environment by building predictive models of the world, which in turn fuel cognitive processes such as directed exploration, goaldirected decisions and forward planning ${ }^{1-3}$. While these internal models can take diverse mathematical forms, their efficiency always depends on the use of task-relevant and cost-efficient state spaces ${ }^{4-6}$. Most often, these state spaces are actually state-action spaces in which the actions of the agent actively contribute to the prediction of upcoming events. For example, a driver must take into account the movement of their hands in order to predict the future position of their car. By contrast, a passenger worried for their safety should ignore their own hands and instead focus on the hands of the driver in order to anticipate potential hazards.

Determining whether an environment is controllable or not is key to decide to which extent one's actions should influence the prediction process, since only controllable environments afford causal influence over state transitions. Controllable contexts thus prompt the use of "actor" models including one's own actions as predictors, whereas uncontrollable contexts prompt the use of simpler "spectator" models linking past and future states of the environment. By gating the causal influence of action selection, controllability likely plays a central role in the engagement of elaborate action selection mechanisms. Supporting this idea, it is well established that prior exposure to controllable contexts promotes proactive and goal-directed strategies in a variety of cognitive tasks ${ }^{7,8}$. Conversely, the lack of perceived control over events, especially stressful ones, constitutes a well-established correlate and a potential predictor of prevalent psychiatric disorders involving an increased influence of reactive and habitual behaviours, such as depression, anxiety, post-traumatic stress or obsessive-compulsive disorders ${ }^{9-13}$.

Numerous studies have shown that exposure to uncontrollable stressors can induce a state of learned helplessness characterized by the generalization of passive reactions to subsequent challenges ${ }^{7,8}$. Evidence indicates that this maladaptive state largely depends on functional changes within the medial prefrontal cortex (mPFC) and the serotonin system ${ }^{14-18}$. In humans, a handful of neuroimaging experiments have further suggested that the anterior insula and cingulate cortex contribute to the detrimental effects of uncontrollable stressors ${ }^{19,20}$. Beyond stress induction 
studies, the sense of being in control of one's own actions and their outcomes is known to modulate hemodynamic responses parietal and prefrontal cortices ${ }^{21-23}$ and the right temporoparietal junction (TPJ) was found to track the divergence of action-outcome transitions, a feature of controllable environments ${ }^{24}$.

Yet, little is known about the algorithms by which the brain estimates dynamically to what degree a task is controllable. A general strategy is to estimate controllability by computing the causal effect the agent's own actions have over the environment. Formally, a task can thus be deemed controllable when the transfer entropy (TE) - a generalization of Granger causality to non-linear and discrete systems - linking state and action time-series is positive ${ }^{25,26}$. By comparing the entropy of observed states given previous states and actions $\left[\mathrm{H}\left(\mathrm{S}^{\prime} \mid \mathrm{S}, \mathrm{A}\right)\right]$ to the entropy of observed states given previous states only $\left[\mathrm{H}\left(\mathrm{S}^{\prime} \mid \mathrm{S}\right)\right]$, this information-theoretic quantity isolates the effective causal influence of actions over state transitions (Fig. 1a, Supplementary Note). Here, we develop a computational model that tracks a dynamic approximation of TE and we use it to shed light on the cognitive and neural mechanisms supporting the ability to infer task controllability and adapt behaviour accordingly.

Based on this information-theoretic formalism, we designed an explore-and-predict task that allowed us to manipulate task controllability and assess the resulting changes in terms of subjective controllability and prediction accuracy. This new task was first used in behavioural $(\mathrm{n}=50)$ and fMRI ( $n=32)$ experiments which aimed at: (a) demonstrating that humans infer task controllability by estimating an approximation of transfer entropy; (b) establishing the dissociation of spectator and actor models predicted by the transfer entropy hypothesis at the behavioural and neural levels; and (c) unraveling the neural substrates underlying the representation of controllability itself and its influences on behaviour. In a subsequent stress experiment $(n=54)$, we exposed participants to either uncontrollable or controllable electric shocks before administering the explore-and-predict task in order to (d) provide causal evidence supporting a dissociation of the spectator and actor models and (e) test whether learned helplessness can be characterized by an increased reliance on the former relative to the latter. 


\section{RESULTS}

\section{Experimental paradigm and computational model}

Healthy human participants were invited to explore an abstract environment composed of three states (square, circle, triangle) and three actions (yellow, blue, magenta). A hidden transition rule always determined upcoming states, either dependent on the action of the participants (controllable rules, C) or the previous state only (uncontrollable rules, U) (Fig. 1b). The transition rules were probabilistic and reversed covertly, so participants needed to explore and accumulate evidence in order to tell which rule was operative. From time to time, the participant was asked to predict the most likely upcoming state given a state-action pair (e.g. "blue" action in "circle" state), and its counterfactual (e.g. "yellow" action in "circle" state). This procedure yielded a direct yet implicit assessment of their subjective sense of controllability, because counterfactual predictions should only differ in controllable contexts, where selected actions determine upcoming states (Fig. 1c). A novel and distinguishing feature of our task is that controllability varied independently of uncertainty (Fig. 1d), a methodological improvement over earlier paradigms where the two constructs covary systematically ${ }^{13,24,27}$. Another key difference from previous studies is that we did not include any reinforcers: participants were merely instructed to explore their environment with the goal of performing accurate predictions when asked to. Here, controllability estimation can interact with, but does not depend on reward and punishment processing ${ }^{7,28,29}$, the only requirement being to maintain a minimal level of exploration, or noise in the action selection process (Fig. 1e, Supplementary Text).

To untangle the mechanisms of controllability estimation in this task, we designed a computational architecture for dynamically tracking an approximation of TE (for a detailed description, see Supplentary Fig. 1a, Methods). Paralleling the standard computation of TE, two sets of transition probabilities were monitored, one corresponding to an "actor" model (tracking state-action-state transition, SAS') and the other to a "spectator" model (tracking state-state transition, SS', Fig. 1f,i). Following each transition, an approximation of TE (hereafter termed $\Omega$ ) was updated in proportion to the difference between 'actor' and 'spectator' transition probabilities psas'-pss' (Fig. 1f,ii). Intuitively, this difference term can be understood as an instantaneous causality signal, 
reflecting how likely the last state transition towards S' was due to the influence of action A rather than state S. By integrating pSAS'-psS' over time, $\Omega$ thus reflects the causal influence of actions on recent state transitions (Supplementary Fig. 1b).

This causality signal $\Omega$ is at the core of the proposed algorithm, which arbitrates between the actor and the spectator model when making predictions about upcoming states. Specifically, the relative weight of the actor versus spectator model is set by an arbitrator (hereafter termed $\omega$ ) whose value can be interpreted as an estimate of controllability. Two parameters influence the mapping between $\Omega$ and $\omega$ : a threshold determining how much causal evidence is required to infer controllability and a slope determining how fast controllability estimates change around that threshold (Fig. 1f,iii). This SAS'-SS'- $\Omega$ algorithm was contrasted with a conventional model-based reinforcement learning algorithm monitoring SAS' transitions, as implemented by the actor model alone ${ }^{2,30}$. Importantly, this simpler algorithm could still learn transition probabilities from both uncontrollable and controllable conditions in stable environments, but the lack of controllabilitydependent arbitration makes it less efficient in volatile environments alternating rapidly between controllable and uncontrollable rules.

\section{Flexible adjustment of behaviour to changes in controllability}

Participants performed well on the task: in all experiments, the average prediction accuracy was substantially above chance [chance level: 1/3; behavioural: t(49)=12.3, p<0.001; fMRI: $\mathrm{t}(31)=13.4, \mathrm{p}<0.001$; stress: $\mathrm{t}(53)=13.2, \mathrm{p}<0.001]$ (Fig. 2a). In the fMRI experiment, for which participants received more training, accuracy was also stable across conditions and time (Supplementary Table 1). Prediction accuracy dropped and then rapidly recovered after covert reversals in transition rules: it already exceeded chance levels on the first prediction trial after reversal for all types of reversals (all t(49) $>5.38$ and all $p<0.001]$ (Fig. 2b). Prediction accuracy also correlated positively with working memory capacity as indexed by d-primes in a standard 2back task [U rules: $\rho=0.37, p=0.006$; $C$ rules: $\rho=0.52, p<0.001]$ (Fig. 2 c), consistent with the engagement of a model-based learning process ${ }^{31}$. 
In line with our prediction that humans solve the task by estimating $\Omega$, Bayesian model comparisons demonstrated that SAS'-SS' $-\Omega$ schemes outperformed the conventional model-based learning algorithm (SAS' alone) in all experiments (Fig. 3a; Supplementary Fig. 2a), Simulation analyses confirmed that the model was identifiable and that its parameters could be recovered accurately (Supplementary Fig. 2b-d). As expected, the arbitrator $\omega$ captured quantitative changes in subjective controllability, indexed by the proneness of participants to predict that different actions would lead to different states in counterfactual prediction trials (Fig. 3b). Critically, the SAS'-SS'- $\Omega$ scheme which included an arbitration mechanism accounted better for the dynamics of subjective controllability changes around reversals than did the SAS' model alone (Fig. 3c, correct prediction of controllability: $72.7 \%$ versus $66.5 \%, z(49)=4.69, p<0.001$ ). The benefits of monitoring controllability is further illustrated by the finding that the likelihood of using the SAS'-SS'- $\Omega$ scheme over the SAS scheme increased with accuracy across subjects [behaviour: $\mathrm{r}=0.58, \mathrm{p}<0.001$; $\mathrm{fMRI}$ : $\mathrm{r}=0.32, \mathrm{p}=0.07$; stress: $\mathrm{r}=0.54, \mathrm{p}<0.001$ ] (Supplementary Fig. 2e-g).

\section{Behavioural and neural dissociation of the actor and spectator models}

Model comparison results are consistent with our proposal that subjects estimate the subjective controllability of an environment by separately tracking and comparing an actor and a spectator model. In order to further test the dissociation of the actor and spectator models, we used subjectlevel GLMs to assess trial-by-trial fluctuations of decision times. It is known that decision times slow down following state prediction errors ${ }^{32,33}$. The large amount of exploratory trials per participant thus allowed us to analyze decision times as a proxy of model updating and to evaluate to which extent controllability per se influences the speed of action selection [behaviour: $562+/-$ 163 trials; fmri: 550+/-115; stress: 519+/-84]. We therefore extracted the prediction errors derived from both the actor and spectator models (hereafter termed $\delta_{S A S}$, and $\delta_{S S}$ ). We found that both type of prediction errors slowed responding [behavioural: betas $\delta_{\mathrm{SAS}}: \mathrm{t}(49)=3.53, \mathrm{p}<0.001$; betas $\delta_{\mathrm{SS}}$ : $\mathrm{t}(49)=7.93, \mathrm{p}<0.001$; fMRI: betas $\delta_{\text {SAS }}: \mathrm{t}(31)=3.50, \mathrm{p}=0.001$; betas $\left.\delta_{S S^{\prime}}: \mathrm{t}(31)=2.46, \mathrm{p}=0.020\right]$ (Fig. 3e) and independently explained variance in decision times (Supplementary Figure 3). We also observed that in periods of higher estimated environmental controllability (i.e. higher $\omega$ ), decision times were slower [behavioural: $\mathrm{t}(49)=2.20, \mathrm{p}=0.032$; $\mathrm{fMRI}: \mathrm{t}(31)=3.78, \mathrm{p}<0.001$ ]. This effect 
suggests that controllable contexts promote a more controlled action selection process even when no reinforcement is at stake.

Separable neural correlates should therefore exist for the prediction errors generated by the actor and spectator probability tracking processes, $\delta_{\text {SAS }}$, and $\delta_{S S}$ '. A conjunction analysis first revealed that both types of prediction errors activated the typical set of bilateral brain areas commonly associated with state prediction errors ${ }^{2,30}$, such as the frontoparietal network and the presupplementary motor area (Fig. 4a, Supplementary Table 2). When contrasting events where only $\delta_{\mathrm{SS}}$, or only $\delta_{\mathrm{SAS}}$ ' were above their median value (a contrast circumventing collinearity issues), the mPFC significantly dissociated the two prediction error terms (Fig. 4b, Supplementary Table 3, Supplementary Fig. S4a-b for robustness checks). Mixed-effects ROI analyses indicated that the $\mathrm{mPFC}$ encoded $\delta_{\mathrm{SS}}$ ' more negatively than $\delta_{\mathrm{SAS}}$.

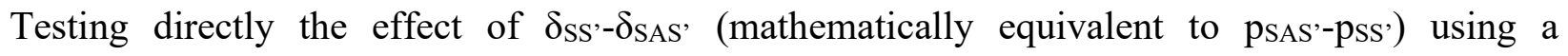
conventional parametric analysis at the whole brain level showed that the mPFC also negatively encoded this signal required for the update of controllability. Like the mPFC, the nucleus accumbens encoded $\delta_{\text {SS }}$ - $\delta_{\text {SAS }}$, negatively, but ROI analyses indicated that this effect stemmed from a positive response to $\delta_{\text {SAS }}$, and an absence of relationship with $\delta_{S S}$, (Fig. 4c, Supplementary Table 3). Interestingly, a similar pattern was observed in the dopaminergic nuclei of the brainstem at a more lenient threshold (Supplementary Fig. 4C).

\section{Neural correlates of dynamic controllability}

Having established the dissociation of $\delta_{\text {SAS' }}$ and $\delta_{\text {SS }}$, at the behavioural and neural levels, we next probed the correlates of the prediction error $\delta_{\Omega}$ governing changes in estimated controllability $\left(\delta_{\Omega}=\delta_{\mathrm{SS}},-\delta_{\mathrm{SAS}}, \Omega_{\mathrm{t}-1}\right)$. This second order learning mechanism is key to accumulate, over time, evidence in favor or against the controllability of the ongoing rule. Whole-brain analyses revealed a significant negative relationship between $\delta_{\Omega}$ and neurovascular responses in the posterior cingulate (PCC, BA 29/30) and dorsal posterior cingulate cortex (dPCC, BA 23/24), the right dorsal anterior insula (dAI) and the right temporo-parietal junction (TPJ, Figure 4d, Supplementary Table 4). Mixed-effects ROI analyses including decision times and $\Omega$ confirmed 
that these effects reflected a genuine response to controllability prediction error, peaking 4-8 seconds after trial onset. Interestingly, we observed a controllability-dependent coupling between the PCC and the mPFC cluster found to encode the difference term $\delta_{S S}$ '- $\delta_{\text {SAS }}$, (Supplementary Fig. 4d). Moreover, interindividual variability in the proneness to rely on the actor model and to perceive the environment as controllable was partly explained by the strength of $\delta_{\Omega}$ encoding in this structure [r=-0.47, p=0.007](Fig. 4e, Supplementary Fig. 4e-f).

In order to unravel the neural correlates of controllability with maximal sensitivity, we performed a multivoxel pattern analysis (MVPA). A support vector machine classifier was trained at predicting whether streaks of consecutive exploratory trials were governed by controllable or uncontrollable rules. Whole-brain maps of classification accuracy were obtained using the searchlight method (leave-one-run-out cross-validation). Objective rule controllability could be decoded above chance from most regions of the frontoparietal control network. Local patterns of activity in the precuneus, the right TPJ, the dlPFC (bilaterally) and the dorsomedial prefrontal cortex (dmPFC) were all sensitive to controllability (Fig. 4f, Supplementary Table 5). Interestingly, the sensitivity of the dmPFC to controllability predicted to which extent controllable contexts lengthened decision times from one participant to another $[\mathrm{r}=0.53, \mathrm{p}=0.002](\mathbf{F i g} . \mathbf{4 g})$.

\section{Uncontrollable stressors promote reliance on the spectator model}

We applied this new paradigm to better understand the computational mechanisms underlying learned helplessness. More precisely, we hypothesized that exposure to uncontrollable stressors might bias controllability estimation mechanisms to promote reliance on the spectator model relative to the actor model. We invited participants to perform an active avoidance task exposing them to mild electric shocks prior to completing the explore-and-predict task (Fig. 5a). Participants in the controllable group learned to avoid the shock following one of the three possible cues by pressing the correct response button (out of six alternatives). Shocks received by participants in the uncontrollable group were yoked to the former, so that their decisions had no influence on shock probability. As expected, this procedure induced a dissociation between actual shock frequency, matched across groups by design, and reported shock expectancy (Fig. 5b), so that shock expectancy remained high until the end of the induction phase in the uncontrollable group. 
Despite the absence of aversive reinforcers in the explore-and-predict task, we observed clear carry-over effects from the shock experiment when analyzing the model parameters governing controllability estimation (Fig. 5c, Supplementary Table 6). In particular, the threshold parameter increased significantly in the uncontrollable group compared with the controllable group $[\mathrm{t}(52)=2.82, \mathrm{p}=0.007]$. This parameter determines how much causal evidence is required before controllability is inferred. Therefore, when making predictions, participants exposed to uncontrollable stressors relied more on the spectator model, demonstrated by the reduced average value of the arbitrator $\omega[\mathrm{z}=-2.44, \mathrm{p}=0.015]$ as well as the direct analysis of subjective controllability estimates, revealing that counterfactual predictions were more often identical in the uncontrollable group $(\mathrm{z}=1.69, \mathrm{p}=0.045$, one-tailed $)$. Importantly, counterfactual predictions did not differ during the training phase, which occurred before stress induction $[\mathrm{z}=-0.58, \mathrm{p}=0.56]$.

Exposure to uncontrollable stressors thus elicits an imbalance between actor and spectator mechanisms for transition probability learning consistent with a sustained shift in controllability expectations. This finding provides a parsimonious account of the cross-contextual generalization of passive strategies, a core feature of helpless states ${ }^{7,8}$. Interestingly, state anxiety, as assessed before the experiment, moderated the induction of controllability estimation biases. It predicted the average value of the arbitrator only in participants exposed to uncontrollable stressors [U: $\mathrm{r}=$ 0.47, $p=0.014 ; C: r=0.24, p=0.21$, U versus $C: z=-2.59, p=0.01]$ (Fig. 5d).

Since uncontrollable stressors promoted increased reliance on the spectator model and decreased reliance on the actor model, we expected PE-dependent slowing effects to follow a similar pattern. Confirming this prediction, the type of stress induction profoundly altered the slowing of decision times by actor and spectator prediction errors [interaction group by PE type: F(1,52)=9.34]. In the uncontrollable group, $\delta_{\mathrm{SAS}}$ ' no longer modulated decision times whereas the impact of $\delta_{\mathrm{SS}}$, was increased compared with the controllable group $\left[\delta_{\mathrm{SS}}: \mathrm{t}(52)=2.08, \mathrm{p}=0.042 ; \delta_{\mathrm{SAS}}\right.$ : $\mathrm{t}(52)=-$ $3.37, \mathrm{p}=0.001]$. Similarly, $\Omega$ no longer predicted decision times $[\mathrm{t}(26)=1.04, \mathrm{p}=0.15]$, contrary to what was observed in the controllable group $[\mathrm{t}(26)=1.98, \mathrm{p}=0.029]($ Fig. 5e) and in the other two experiments. 


\section{DISCUSSION}

Taken together, these findings shed light on one of the most fundamental aspects of human experience: the ability to estimate to which extent our actions affect our environment and to adjust our decisions accordingly. Our results demonstrate that this ability involves the comparison of actor and spectator models of the ongoing task, which are dissociable computationally, behaviourally and neurally. In turn, controllability estimates can be used to arbitrate between these models when making predictions about future events. The mPFC and the striatum encode the difference between the prediction errors generated by each model, while signals related to the update of controllability estimates are found in a more posterior brain network encompassing the TPJ and the PCC. Furthermore, exposure to uncontrollable stressors biases this process assessed by the explore-and-predict task, hence establishing its relevance for the study of neuropsychiatric disorders involving altered perceptions of controllability $9,12,13,34$.

Historically, the concept of task controllability has been heavily influenced by learned helplessness studies in which animals granted the ability to actively terminate stressors are compared to yoked animals exposed to the exact sequence of stressors, but whose actions are made independent from stressor termination ${ }^{8,14,15}$. In this line of research, focused on the long-lasting consequences of stress exposure, more controllable contexts were defined as those in which the mutual information linking the timings of actions and stressor offsets is higher ${ }^{35}$. However, a positive mutual information linking an organism's actions and upcoming states of the environment is a necessary but not a sufficient condition to declare a task controllable. For example, the highly positive mutual information linking the statements of a weather forecaster with the occurrence of rain should obviously not be interpreted as a sign that the forecaster controls the weather, because the statements of the forecaster and the occurrence of rain are both conditioned by past meteorological states. Moreover, following this incomplete definition, variations of task controllability were often obtained by manipulating uncertainty about future states ${ }^{17,20,27}$, hence leading to ambiguous conclusions regarding the mechanisms underlying the estimation of controllability per se and its downstream influence on behaviour. 
Formalizing controllability using transfer entropy (TE) rather than mutual information allowed us to design a task in which controllability varied independently from uncertainty. In addition, this approach provided an algorithm for detecting genuine changes in task controllability. Model comparisons showed that, across the three experiments, algorithms monitoring controllability using an approximation of TE (i.e SAS'-SS'- $\Omega$ schemes) accounted better for participants' choices than a standard model-based learning algorithm. The analysis of reaction times confirmed that participants were sensitive to the prediction errors generated by the spectator and actor models. These two first-order models, whose comparison governed the update of controllability estimates, can be viewed as two state spaces competing to structure the learning of statistical contingencies. When controllability estimates are low, the spectator model representing only the successive states of the environment dominates. In contrast, when controllability estimates are high, the actor model representing both states and actions takes over.

By defining the most appropriate state space dynamically, controllability estimation improves predictions about the future states of one's environment and can therefore contribute to maximize utility when reward or punishment rates depend on such predictions. By promoting reliance on a simpler spectator model when the environment is deemed uncontrollable, it can also minimize the metabolic cost and subjective effort associated with controlled action selection ${ }^{36,37}$. These hypotheses could be tested directly by introducing reinforcers in the explore-and-predict task, but it is already worth to note that the controllability-dependent arbitration logics can readily explain why Pavlovian (equivalent to SS') and instrumental (equivalent to SAS') learning mechanisms are respectively promoted uncontrollable and controllable contexts ${ }^{27}$. Restricted to the striatum and the mPFC, the limited spatial dissociation of the actor and spectator models reported here is consistent with a recent MEG study showing that the human brain relies on shared "neural codes" to infer hidden states in controllable and uncontrollable contexts ${ }^{38}$. Yet, signatures of the actor and spectator models may ultimately be found within local neural circuits, which are beyond the reach of standard fMRI and MEG techniques. Moreover, the preferential encoding of actor prediction errors by the striatum and dopaminergic midbrain is consistent with earlier findings showing that the mesolimbic pathway preferentially encodes reward prediction errors in instrumental learning tasks $^{6,39-41}$. Conversely, the preferential encoding of spectator prediction errors by the mPFC is consistent with its more general role in statistical learning ${ }^{42,43}$. It may explain why dysfunctions of 
this structure impair observational learning more profoundly than instrumental learning ${ }^{44,45}$ and why mPFC lesions can alter the perception of controllability without altering performance of simple instrumental learning tasks ${ }^{46}$.

By comparing the predictions emanating from the actor and spectator models, one can derive an instantaneous causality signal (i.e how likely did the last action cause the last state transition). Encoded negatively by mPFC and striatal BOLD responses, this instantaneous signal can then be integrated over time, hence reflecting the causal influence of actions over recent transitions. A signature of the second-order prediction errors supporting this integration was found in the right TPJ, right insula, PCC and APCC. The right TPJ was the only region sensitive to both these secondorder prediction errors as well as to objective controllability as assessed by the decoding analysis. It is thus a strong candidate for the implementation of controllability monitoring in our task. Supporting this view, the right — but not left — TPJ has previously been found to encode the divergence in action-outcome distributions ${ }^{24}$ and the discrepancy between expected and actual outcome timings in a simple sensorimotor task alternating controllable and uncontrollable trials ${ }^{23}$. Other brain areas including the dlPFC and the dmPFC were sensitive to objective controllability, likely reflecting downstream adaptations of brain networks to task controllability ${ }^{47}$. Strikingly, a higher sensitivity of the dmPFC to controllability was associated with a stronger influence of controllability on decision times. This finding suggests that controllability detection fosters more elaborate action selection processes by potentiating a form of proactive response inhibition previously linked to dmPFC activity ${ }^{48}$. It might be noted that the dmPFC region discussed here is more dorsal than the AACC region previously implicated in the valuation of control ${ }^{49}$.

Interestingly, the PCC exhibited a controllability-dependent coupling with the mPFC and participants with a more (negative) encoding of controllability prediction errors in the PCC had a higher propensity to rely on the actor model when making predictions. Based on this finding, we may speculate that deactivations of the PCC facilitate the switch towards the actor model whenever the environment is deemed controllable. Supporting this view, electrical stimulation of the human PCC can elicit behavioural idleness and loss of control feelings ${ }^{50}$, a phenomenon possibly due to the sudden blunting of action monitoring mechanisms ${ }^{51}$. Future studies may test whether the 
heightened metabolic activity of the $\mathrm{pCC}^{52}$ and its unstable connectivity with the mPFC during major depression ${ }^{53}$ contributes to the lower sense of control observed in this condition ${ }^{9}$.

Having described the computational principles and outlined neural correlates of controllability estimation, we sought to test whether an experimental manipulation could alter this process and simultaneously contribute to a better understanding of the learned helplessness phenomenon. Indeed, exposure to uncontrollable stressors is known to induce passive responses to subsequent controllable stressors, but the origins of this maladaptive strategy remain poorly understood. In particular, it is unclear whether prior exposure to uncontrollable stressors induces an increased sensitivity to future aversive events, reduces expectation of control with respect to future stressors or reduces expectations of control in general ${ }^{8,28}$. Our results support the latter hypothesis by showing sustained alterations of controllability estimation in human participants previously exposed to uncontrollable versus controllable stressors. More precisely, the specific increase observed for the threshold parameter implies that the former group needed to integrate more causal evidence before considering a given rule as controllable in the explore-and-predict task. The dorsal anterior insula $(\mathrm{dAI})$ is involved in the modulation of pain perception by controllability ${ }^{20}$ and it was found to encode controllability prediction errors in our fMRI experiment. Therefore, it is possible that prior exposure to uncontrollable stressors altered dAI excitability to distort subsequent controllability estimation mechanisms. Supporting this idea, a study showed that a lower perception of control mediates the exacerbation of dAI responses to physical threats in more anxious individuals, who also displayed lower controllability estimates following uncontrollable stressors in our data ${ }^{19}$. Yet, this increased reliance on the spectator relative to the actor model following uncontrollable stressors likely involves several other brain areas, including the mPFC and the dorsal raphe nucleus, both highly sensitive to stressor controllability ${ }^{14-16}$.

In sum, the explore-and-predict task allowed us to isolate the core computations supporting the inference of task controllability by excluding reinforcers and matching uncertainty across contexts. By showing that the human brain can compute an approximation of transfer entropy, our study may help bridging the gap between neuroscience and artificial intelligence research, where transfer entropy plays an important role in solving unsupervised learning problems ${ }^{54,55}$. More invasive techniques will be required to understand how these computations are implemented within local 
neural circuits and how neuromodulators such as dopamine or serotonin mediate their broad impact on stress responses and mental health ${ }^{7,14,15,56}$.

\section{METHODS}

\section{Participants}

For the behavioural experiment, fifty young adult participants (mean age: 24.7, range: 18—43, 27 women) were recruited via the Sona system (human subject pool management system) of the Radboud University (The Netherlands). All participants were included in the data analysis. For the fMRI experiment, thirty-two young adult participants (mean age: 25.1 , range: 20 — 43, 18 women) were recruited through the same system. For the stress experiment, a total of 62 participants (mean age $=21.8$; range: $18-27,52$ women) were recruited via the Sona system of Leiden University. One additional participant was excluded a posteriori from the fMRI experiment and four participants were excluded from the stress experiments, together with their yoked counterparts (see Supplementary Methods for details on exclusion and inclusion criteria). The behavioural and fMRI experiments were approved by the local ethics committee (CMO region Arnhem/Nijmegen, The Netherlands, CMO2001/095). The stress experiment was approved by the Psychology Research Ethics Committee (CEP17-0905/282) at Leiden University. All participants provided written informed consent, in line with the declaration of Helsinki.

\section{Explore-and-predict task}

In the 3 experiments, the overall structure of the task was identical. Participants performed 6 (fMRI and stress experiment) or 7 (behavioural experiment) exploratory trials before a pair of predictions was required. Pairs of predictions always probed the two actions available for a given state (e.g blue followed by yellow in the circle state), in order to derive subjective control lability from counterfactual responses. Participants received feedback about their predictions in 50 percent (fMRI and stress) or 100 percent (behavioural experiment) of the trails. In the fMRI and stress 
tasks, feedback was delivered only after one of the two counterfactual predictions in order to prevent participants from inferring whether the rule was controllable or not based on feedback.

On each exploratory trial, two identical geometrical shapes were displayed side by side. The color of each shape determined the action corresponding to left and right button presses (side randomly assigned in each trial). An urgency signal was displayed after $1.5 \mathrm{~s}$. Transitions to the next state were always governed by one of the four rules, as displayed in Fig. 1c. In order to maximize the variation of prediction errors, the transitions were stochastic (noise: 0.05 to 0.2 ).

The first prediction trial of each pair was simply displayed at the end of the ITI of the previous exploratory trial. An urgency signal was displayed after 4s. The hypothetical state action pair was displayed at the center of the screen, just below a question mark, and the 3 possible next states were displayed as white geometrical shapes at the top of the screen. The selected state was then highlighted and the feedback was displayed when applicable.

The ongoing rule was never changed before 4 pairs of predictions were completed. In the behavioural experiment, the rule changed from then as soon as 5 correct responses were provided in the last 6 predictions or if the last 4 predictions were accurate. In the fMRI experiment, the rule was changed as soon as the p-value of a binomial test indicated that accuracy was significantly below chance $(p<0.05$, one-tailed, chance level: $1 / 3)$, hence making the accuracy threshold more lenient as the number of predictions made for a given rule increased. In all experiments, the rule changed after 10 pairs of predictions, even if performance did not meet the learning criterion. Prediction trials were pseudo-randomly ordered with the constraint that each state would be tested a similar number of times. An exhaustive description of instructions, counterbalancing, reversal schedules, transition noises and timings is available in Supplementary methods.

\section{Stress induction task}

To test the impact of prior controllability over stress on subsequent controllability estimations, participants underwent a stressor controllability manipulation prior to the explore-and prediction task in a between-subjects design. Critically, we employed a between-subjects yoked control 
procedure in order to match the amount and order of aversive outcome stimuli between the controllable and uncontrollable conditions. We randomized participants in blocks of four where the controllable condition of a yoked pair was always administered first in order to create the schedule for the yoked counterpart in the uncontrollable condition.

Electric stimuli served as stressors in the manipulation task and were delivered by a Digitimer DS7 stimulator. First, individual levels of intensity of the electric stimulus for the manipulation task were determined using a stepwise procedure in which the intensity of the stimulus was gradually increased until participants reported a 'just bearable, but not yet painful' experience of shock. A yoked control-design with preprogrammed pseudorandomized schedule enabled us to match the amount and order of electric stimuli between the conditions.

In the controllable condition, a total of four cues (different in shape and color) were presented for at least six repetitions each following a preprogrammed pseudorandomized schedule. Participants could learn by trial-and-error the correct response corresponding to the cue (a key between 1 and 6) to avoid the electric stimulus. Critical trials on which participants would be able to prevent the electric stimulus for the first time according to the schedule were repeated until the participants arrived at a correct response. As such, all participants underwent the whole schedule with a minimum of 24 trials, and were able to acquire the correct response for each cue.

The uncontrollable condition was yoked to the controllable condition, such that participants experienced a comparable pattern of events across conditions. However, in the uncontrollable condition, participants were not able to acquire these action-outcome contingencies to prevent the shocks, whose sequences were merely replayed from the yoked participants performing the controllable condition. An exhaustive description of counterbalancing, instructions and procedures is available in Supplementary methods.

\section{Computational modeling}

The main purpose of all SAS'-SS' $\Omega$ algorithms is to provide a way to dynamically estimate the causal influence of actions over state transitions by updating a variable termed $\Omega$. In all models, $\mathrm{S}$ 
represents the previous state of the environment, A represents the previous action and S' represents the current state of the environment. The local causality estimate $\Omega$ can only be used as a proxy for controllability, which is not a property of actions but of the environment. It is this "inferred controllability" variable, termed $\omega$, which can then be used to decide (arbitrate) whether one should make predictions using learned S-S' transitions or learned SA-S' transitions. $\Omega$ is homologous to transfer entropy (TE, which is itself a generalization of Granger causality to discrete and non-linear domains). See Supplementary Methods for a detailed explanation of the differences between TE and $\Omega$.

In order to demonstrate that participants used a dynamic estimate of transfer entropy to solve the task, we systematically compared variants of the SAS'-SS' $-\Omega$ algorithm to a standard model based architecture tracking SAS' transitions ${ }^{2}$. This latter algorithm corresponds to the actor model alone. Its asymptotic performance in stable environments is identical to that of SAS'-SS' $\Omega$ algorithms.

The actor model tracks transitions linking state-action pairs to newly encountered states (i.e. SAS'). It updates transition probabilities in the following fashion.

Realized transitions:

$$
P\left(s^{\prime} \mid s, a\right) \leftarrow P\left(s^{\prime} \mid s, a\right)+\alpha\left(1-P\left(s^{\prime} \mid s, a\right)\right)
$$

Unrealized transitions:

$$
P\left(s^{\prime} \mid s, a\right) \leftarrow P\left(s^{\prime} \mid s, a\right)(1-\alpha)
$$

Where $\alpha \in[0,1]$ controls to which extent learned transition probabilities are determined by the most recent transitions. The prediction error $1-\mathrm{P}\left(\mathrm{s}^{\prime} \mid \mathrm{s}, \mathrm{a}\right)$ is noted $\delta_{\mathrm{SAS}}$, in the main text.

The spectator model tracks transitions linking states to newly encountered states (i.e. SS'). Therefore, it updates transition probabilities exactly like the actor model, except that only states are represented: $\mathrm{P}\left(\mathrm{s}^{\prime} \mid \mathrm{s}, \mathrm{a}\right)$ is simply replaced by $\mathrm{P}\left(\mathrm{s}^{\prime} \mid \mathrm{s}\right)$ in the two equations above, and the prediction error $1-\mathrm{P}\left(\mathrm{s}^{\prime} \mid \mathrm{s}\right)$ is noted $\delta_{\mathrm{ss}}$, in the main text.

The variable $\Omega$ supports the controllability estimation process by tracking the expected differ ence $\mathrm{P}\left(\mathrm{s}^{\prime} \mid \mathrm{s}, \mathrm{a}\right)-\mathrm{P}\left(\mathrm{s}^{\prime} \mid \mathrm{s}\right)$ dynamically (or, equivalently, $\left.\delta_{\mathrm{SS}}{ }^{\prime}-\delta_{\mathrm{SAS}}\right)$. The logic of this process is that, in a controllable environment, actions contribute to predicting the upcoming states and therefore $\mathrm{P}\left(\mathrm{s}^{\prime} \mid\right.$ 
$\mathrm{s}, \mathrm{a})>\mathrm{P}\left(\mathrm{s}^{\prime} \mid \mathrm{s}\right)$. Higher values of $\Omega$ therefore imply higher evidence that the environment is controllable. The update of $\Omega$ is governed by the following equation:

$$
\Omega \leftarrow \Omega+\alpha_{\Omega}\left(P\left(s^{\prime} \mid s, a\right)-P\left(s^{\prime} \mid s\right)-\Omega\right)
$$

Where $\alpha \Omega \in[0,1]$ is the learning rate controlling to which extent $\Omega$ is determined by the most recent observations.

Since $\Omega$ reflects the causal influence of one's action over state transition, it can be used as a proxy to infer whether the environment is likely controllable or uncontrollable. In order to form the arbitration term reflecting this inference and accommodate inter individual differences at this step, $\Omega$ is thus transformed using a parametrized sigmoïd function:

$$
\omega=\frac{1}{1+\exp \left(-\beta_{\Omega}\left(\Omega-\text { thres }_{\Omega}\right)\right.}
$$

Where thres $\Omega \in[-1,1]$ corresponds to the threshold above which $\Omega$ is interpreted as evi dence that the environment is controllable and where $\beta_{\Omega} \in[0$,Inf $]$ determines to which extent evidence that the environment is controllable (i.e. $\Omega-$ thres $\Omega>0$ ) favors reliance on learned SAS' transitions when making predictions (and vice-versa for SS' transitions when $\Omega-$ thres $\Omega<0$ ). Thus, the variable $\omega$ implements the arbitration between the "actor" and the "spectator" model.

When only SAS' learning is considered, the probability that a given state $\mathrm{S}^{\prime}=\mathrm{i}$ will be observed given $\mathrm{S}$ and $\mathrm{A}$ is directly given by:

$$
p\left(S^{\prime}=i\right)=p\left(S^{\prime}=i \mid S, A\right)
$$

When the SS'-SAS'- $\Omega$ architecture is used, the probability that a given state $S$ ' $=\mathrm{i}$ will be observed given $\mathrm{S}, \mathrm{A}$ and $\omega$ is directly given by:

$$
p\left(S^{\prime}=i\right)=\omega \max _{j=1: 3} p\left(S^{\prime}=i \mid S_{j}, A\right)+(1-\omega) p\left(S^{\prime}=i \mid S\right)
$$

The max operation reflects the fact that participants are explicitly instructed that, in this version of the explore-and-predict task, their actions have the same consequences independently of the state in which they are. Thus, it is reasonable to expect that, under the hypothesis that the environment is controllable, participants will select the most likely transition independently of the state in which they are. Obviously, this step cannot be implemented if only SAS' learning is used, as the model would then lose the ability to discriminate amongst different states. 
The probability that the participant predicts the next state would be i (e.g. a square) when confronted to the hypothetical state-action pair S,A (e.g. circle state, blue action) is given by:

$$
p(\text { prediction }=i)=\frac{\exp \left(\beta_{\text {choice }} p\left(S^{\prime}=i\right)\right.}{\sum_{j=1}^{j=3} \exp \left(\beta_{\text {choice }} p\left(S^{\prime}=i\right)\right)}
$$

Where $\beta_{\text {choice }} \in[-\operatorname{Inf}$, Inf $]$ determines to which extent the participants will systematically select the most likely transition (i.e. the highest $\mathrm{p}\left(\mathrm{S}^{\prime}=\mathrm{i}\right)$, according to what has been learned) to make their predictions. A very positive $\beta_{\text {choice }}$ implies that the participant systematically selects this most likely transition. A $\beta_{\text {choice }}$ around 0 implies that the participant mostly makes random guesses. And a $\beta_{\text {choice }}$ very negative would imply that the participant mostly goes against what he/she has learned.

The full model space was composed of SAS' alone, SAS'-SS'- $\Omega$ balanced and symmetric, SAS'SS' $\Omega$ unbalanced and symmetric, SAS'-SS'- $\Omega$ balanced and asymmetric. SAS'-SS'- $\Omega$ unbalanced and asymmetric. Unbalanced algorithms refer to variants allowing different learning rates for the actor and spectator models. Asymmetric algorithms refer to variants allowing different learning rates for upward and downward updates of $\Omega$. The last 3 algorithms were used to test for imbalances or asymmetries which could contribute to biased perceptions of controllability (e.g. illusion of control), independent from $\Omega$. A full description of these models is available in Supplementary Methods.

\section{Model fitting procedures}

Model fitting was performed using a Variational Bayesian (VB) estimation procedure using the well-validated VBA toolbox ${ }^{57}$. The fitting procedure only attempted to explain decisions made in prediction trials. In other words, the decisions made in exploratory trials only indirectly constrained the fit by determining the information gleaned between pairs of prediction trials. For the behavioural experiments, the prior distributions of the various learning rates and of the threshold parameter were innately defined as Gaussian distributions of mean 0 and variance 3, which approximates the uniform distribution over the interval of interest after sigmoid transformations. The prior distributions of $\beta$ choice and $\beta \omega$ parameters were defined as Gaussian distribution of mean 0 and variance 10. For the fMRI and the stress experiments, the prior distributions of every parameter was defined using the posterior mean and variance obtained from 
the 50 participants who passed the behavioural experiment. Hidden states (i.e values) corresponding to transition probabilities were systematically initialized at 1/3 (equiprobability prior), while $\Omega$ was initialized at 0 . Detailed information about parameter transformation, model fitting, model comparison and simulation procedures is available in Supplementary Methods.

\section{fMRI: acquisition}

All images were collected using a 3T Siemens Magnetom Prismafit MRI scanner (Erlangen, Germany) with a 32-channel head coil. A T2*-weighted multiband echo planar imaging sequence with acceleration factor 8 (MB8) was used to acquire BOLD-fMRI whole-brain covered images $(\mathrm{TR}=700 \mathrm{~ms}, \mathrm{TE}=39 \mathrm{~ms}$, flip angle $=52$, voxel size $=2.4 \times 2.4 \times 2.4 \mathrm{~mm} 3$, slice gap $=0 \mathrm{~mm}$, and FOV $=210 \mathrm{~mm}$ ). This state-of-the-art sequencing protocol was optimized from the recommended imaging guidelines of the Human Connectome Project, with the fast acquisition speed facilitating the detection and removal of non-neuronal contributions to BOLD changes (http://protocols.humanconnectome.org/HCP/3T/imaging-protocols.html).

The experiment was divided in 4 blocks lasting on average 7.7+/-2.1 minutes (662+/-179 volumes). We recorded participants' heartbeats using the scanner's built-in photoplethysmograph, placed on the right index finger. Respiration was measured with a pneumatic belt positioned at the level of the abdomen. Anatomical images were acquired using a T1-weighted MPRAGE sequence, using a GRAPPA acceleration factor of $2(\mathrm{TR}=2300 \mathrm{~ms}, \mathrm{TE}=3.03 \mathrm{~ms}$, voxel size $=1 \times 1 \times 1 \mathrm{~mm}$, 192 transversal slices, $8^{\circ}$ flip angle). Field magnitude and phase maps were also acquired.

\section{fMRI: preprocessing}

fMRI data processing and statistical analyses were performed using statistical parametric mapping (SPM12; Wellcome Trust Centre for Neuroimaging, London, UK). For each session, the first 4 volumes were automatically discarded by the scanner. Functional images were slice-time corrected, unwarped using the field maps and realigned to the mean functional image using a rigidbody registration. Functional images were then coregistered to the anatomical T1. Next, the anatomical images were segmented based on tissue prior probability maps for spatial normalisation 
using the DARTEL algorithm and the resulting normalization matrix was applied to all functional images. Finally, all images were spatially smoothed with a $6 \mathrm{~mm}$ Gaussian kernel, except in the decoding analysis for which unsmoothed images were used.

\section{fMRI analyses}

Statistical analyses of fMRI signals were performed using a conventional two-levels random effects approach in SPM12. All general linear models (GLM) described below included the 6 unconvolved motion parameters from the realignment step. We also included the eigenvariate of signals from cerebrospinal fluid (CSF) in our GLM (fourth and lateral ventricular). Moreover, we used a retrospective image correction (RETROICOR) method to regress out physiological noise, using 10 cardiac phase regressors and 10 respiratory phase regressors obtained by expanding cosines and sines of each signal phase to the 5 th order. We also included time shifted cardiac rates (lag: $+6,+10$ and $+12 \mathrm{~s})$ and respiratory volume $(-1$ and $+5 \mathrm{~s})$ as nuisance regressors. All regressors of interest were convolved with the canonical hemodynamic response function (HRF). All GLM models included a high-pass filter to remove low-frequency artifacts from the data (cut-off $=96 \mathrm{~s}$ ) as well as a run-specific intercept. Temporal autocorrelation was modeled using an AR(1) process. All motor responses recorded were modeled using a zero-duration Dirac function. We used standard voxel-wise threshold to generate SPM maps $(\mathrm{p}<0.001$ uncorrected), unless notified otherwise. All statistical inferences based on whole-brain analyses satisfied the standard multiple comparison threshold $(\mathrm{p}(\mathrm{FWE})<0.05)$ at the cluster level unless notified otherwise. Prediction error and (log-transformed) decision time regressors were systematically z-scored to exclude scaling effects.

All GLM models included separate onset regressors for motor responses, for prediction trials and for the first trial of each exploratory sequence (where no prediction error was elicited). All models also included parametric regressors for reaction time and $\omega$ (reflecting controllability estimates) on prediction trials. A detailed description of the GLMs used to analyze neuroimaging data is available in Supplementary methods. These GLMs only differ in the way exploratory trials were treated. 
In order to verify the robustness of our whole-brain results and inspect the time course of our parametric effects of interest, we performed mixed-effects analyses on BOLD signal filtered and adjusted for nuisance regressors. This adjusted signal was extracted from the functional clusters uncovered by whole-brain analyses and segmented into trial epochs from -3 to +16 seconds around the onset of each exploration trial (excluding the first of each streak). We then estimated the effect of each regressor of interest, at each time point, for all subjects simultaneously. Subject identity was included as a random effect and a subject-specific intercept was included. Parametric regressors were z-scored in the same way as in the mass univariate anal yses. Importantly, this approach was not used for statistical inference - since doing so would constitute double-dipping — but merely for visualization purposes.

Decoding analyses were performed using the TDT toolbox ${ }^{58}$. Each mini-block of 6 exploratory trials was arbitrarily coded as +1 (controllable) or -1 (uncontrollable) based on the rule governing transitions. We used a leave-one-run out cross-validation scheme with 100 permutations per subject, so that classes remained balanced for training. Training was performed on the beta values associated with each miniblock (see previous section) using a Support Vector Machine (SVM) classifier (L2-loss function, cost parameter set to 1, Liblinear, version 1.94), without feature selection or feature transformation. Since we did not constrain the testing sets to have balanced classes, balanced accuracies were used when reporting the results of the searchlight analysis (12mm sphere) at the whole-brain level.

\section{Statistical procedures}

Model selections relied on Bayesian model comparisons and exceedance probabilities, as implemented by the VBA toolbox ${ }^{57}$. The analysis of predictive accuracies relied on a 2-way repeated measure ANOVAs or one-sample t-tests, assuming normal distribution of the data following arcsin transformation. The analysis of decision times was performed in two steps: first, a logistic regression was performed on binarized decision times (median-split) ; second, grouplevel significance was assessed by means of one-sample t-tests. For the analysis of decision times, we excluded trials in which decision times were 3 standard deviations above the mean. Comparison between conditions relied on paired t-tested and comparison between groups (stress experiments) 
relied on two-sample t-tests, unless normality assumptions were violated, in which case nonparametric equivalents were used (Wilcoxon signed rank and rank sum tests, respectively). All ttests were two-sided unless notified otherwise. Correlations were based on Pearson coefficients unless normality assumptions were violated, in which case Spearman rank coefficients were used. Statistical procedures related to the fMRI data are described in the fMRI analysis section above. 


\section{ACKNOWLEDGEMENTS}

We are grateful to Michael Frank for his constructive comments on the manuscript and computational models. We thank Paul Gaalman for his help with fMRI data acquisition. We thank Korina Elefteriadou, Fili Dianellou, Kristin Koelbel, and Julia Breen and SOLO labsupport Leiden University for their help and support in the acquisition of the data for the stress experiment.

Funding: This work was supported by grants from the Fyssen Foundation and the behaviour and Brain Research foundation awarded to RL (Young Investigator 2017) and a Vici award from the Netherlands Organisation for Scientific Research to RC (NWO 453-14-005).

\section{AUTHOR CONTRIBUTIONS}

Conceptualization: RL, RC, VL. Methodology: RL, VL. Software \& formal analysis: RL. Investigation: RL, VL. Resources: RC, ZM. Data Curation: RL, VL. Writing - Original Draft: RL. Writing - Review \& Editing: RL, ZM, VL, RC. Visualization: RL. Funding acquisition: RL, ZM, VL, RC.

\section{DECLARTION OF INTERESTS}

The authors declare no competing interests.

\section{RESOURCE AVAILABILITY}

The behavioural and fMRI data used to generate the figures will be made available upon publication at the following address: https://github.com/romainligneul/controllability. Secondlevel SPM images will be made available upon publication at the following address: https://identifiers.org/neurovault.collection:8810. Raw behavioral and fMRI data will be made available upon request to the corresponding authors.

The scripts used to collect and analyze data will be made available upon publication at the following address https://github.com/romainligneul/controllability. 


\section{REFERENCES}

1. Bastos, A. M. et al. Canonical Microcircuits for Predictive Coding. Neuron 76, 695-711 (2012).

2. Gläscher, J., Daw, N., Dayan, P. \& O’Doherty, J. P. States versus Rewards: Dissociable Neural Prediction Error Signals Underlying Model-Based and Model-Free Reinforcement Learning. Neuron 66, 585-595 (2010).

3. Rao, R. P. N. \& Ballard, D. H. Predictive coding in the visual cortex: a functional interpretation of some extra-classical receptive-field effects. Nat. Neurosci. 2, 79-87 (1999).

4. Niv, Y. Learning task-state representations. Nat. Neurosci. 22, 1544-1553 (2019).

5. Kim, D., Park, G. Y., O'Doherty, J. P. \& Lee, S. W. Task complexity interacts with state-space uncertainty in the arbitration between model-based and model-free learning. Nat. Commun. 10, 5738 (2019).

6. Hamid, A. A., Frank, M. J. \& Moore, C. I. Dopamine waves as a mechanism for spatiotemporal credit assignment. http://biorxiv.org/lookup/doi/10.1101/729640 (2019) doi:10.1101/729640.

7. Moscarello, J. M. \& Hartley, C. A. Agency and the Calibration of Motivated Behavior. Trends Cogn. Sci. 21, 725-735 (2017).

8. Maier, S. F. \& Seligman, M. E. P. Learned helplessness at fifty: Insights from neuroscience. Psychol. Rev. 123, 349-367 (2016).

9. Cheng, C., Cheung, S. F., Chio, J. H. \& Chan, M.-P. S. Cultural meaning of perceived control: A meta-analysis of locus of control and psychological symptoms across 18 cultural regions. Psychol. Bull. 139, 152-188 (2013).

10. Hammack, S. E., Cooper, M. A. \& Lezak, K. R. Overlapping neurobiology of learned helplessness and conditioned defeat: Implications for PTSD and mood disorders. Neuropharmacology 62 , 565-575 (2012).

11. Harrow, M., Hansford, B. G. \& Astrachan-Fletcher, E. B. Locus of control: Relation to schizophrenia, to recovery, and to depression and psychosis - A 15-year longitudinal study. Psychiatry Res. 168, 186-192 (2009).

12. Gillan, C. M. et al. Obsessive-compulsive disorder patients have a reduced sense of control on the illusion of control task. Front. Psychol. 5, (2014).

13. Diener, C., Kuehner, C., Brusniak, W., Struve, M. \& Flor, H. Effects of stressor controllability on psychophysiological, cognitive and behavioural responses in patients with major depression and dysthymia. Psychol. Med. 39, 77-86 (2009).

14. Amat, J. et al. Medial prefrontal cortex determines how stressor controllability affects behavior and dorsal raphe nucleus. Nat. Neurosci. 8, 365-371 (2005).

15. Bland, S. T. et al. Stressor Controllability Modulates Stress-Induced Dopamine and Serotonin Efflux and Morphine-Induced Serotonin Efflux in the Medial Prefrontal Cortex.

Neuropsychopharmacology 28, 1589-1596 (2003).

16. Challis, C. et al. Raphe GABAergic Neurons Mediate the Acquisition of Avoidance after Social Defeat. J. Neurosci. 33, 13978-13988 (2013). 
17. Kerr, D. L., McLaren, D. G., Mathy, R. M. \& Nitschke, J. B. Controllability Modulates the Anticipatory Response in the Human Ventromedial Prefrontal Cortex. Front. Psychol. 3, (2012).

18. Wood, K. H. et al. Controllability modulates the neural response to predictable but not unpredictable threat in humans. Neurolmage 119, 371-381 (2015).

19. Alvarez, R. P. et al. Increased anterior insula activity in anxious individuals is linked to diminished perceived control. Transl. Psychiatry 5, e591-e591 (2015).

20. Bräscher, A.-K., Becker, S., Hoeppli, M.-E. \& Schweinhardt, P. Different Brain Circuitries Mediating Controllable and Uncontrollable Pain. J. Neurosci. Off. J. Soc. Neurosci. 36, 5013-5025 (2016).

21. Haggard, P. Sense of agency in the human brain. Nat. Rev. Neurosci. 18, 196-207 (2017).

22. Kühn, S., Brass, M. \& Haggard, P. Feeling in control: Neural correlates of experience of agency. Cortex 49, 1935-1942 (2013).

23. Spengler, S., von Cramon, D. Y. \& Brass, M. Was it me or was it you? How the sense of agency originates from ideomotor learning revealed by fMRI. Neurolmage 46, 290-298 (2009).

24. Liljeholm, M., Wang, S., Zhang, J. \& O'Doherty, J. P. Neural Correlates of the Divergence of Instrumental Probability Distributions. J. Neurosci. 33, 12519-12527 (2013).

25. Vejmelka, M. \& Paluš, M. Inferring the directionality of coupling with conditional mutual information. Phys. Rev. E 77, (2008).

26. Barnett, L., Barrett, A. B. \& Seth, A. K. Granger Causality and Transfer Entropy Are Equivalent for Gaussian Variables. Phys. Rev. Lett. 103, 238701 (2009).

27. Dorfman, H. M. \& Gershman, S. J. Controllability governs the balance between Pavlovian and instrumental action selection. Nat. Commun. 10, 5826 (2019).

28. Ly, V., Wang, K. S., Bhanji, J. \& Delgado, M. R. A Reward-Based Framework of Perceived Control. Front. Neurosci. 13, 65 (2019).

29. Huys, Q. J. M. \& Dayan, P. A Bayesian formulation of behavioral control. Cognition 113, 314328 (2009).

30. Lee, S. W., Shimojo, S. \& O’Doherty, J. P. Neural Computations Underlying Arbitration between Model-Based and Model-free Learning. Neuron 81, 687-699 (2014).

31. Otto, A. R., Raio, C. M., Chiang, A., Phelps, E. A. \& Daw, N. D. Working-memory capacity protects model-based learning from stress. Proc. Natl. Acad. Sci. 110, 20941-20946 (2013).

32. Shahar, N. et al. Improving the reliability of model-based decision-making estimates in the two-stage decision task with reaction-times and drift-diffusion modeling. PLOS Comput. Biol. 15, e1006803 (2019).

33. Shahar, N. et al. Credit assignment to state-independent task representations and its relationship with model-based decision making. Proc. Natl. Acad. Sci. 116, 15871-15876 (2019).

34. Voss, M., Chambon, V., Wenke, D., Kühn, S. \& Haggard, P. In and out of control: brain mechanisms linking fluency of action selection to self-agency in patients with schizophrenia. Brain 140, 2226-2239 (2017). 
35. Maier, S. F. \& Seligman, M. E. Learned helplessness: Theory and evidence. J. Exp. Psychol. Gen. 105, 3-46 (1976).

36. Westbrook, A. et al. Dopamine promotes cognitive effort by biasing the benefits versus costs of cognitive work. Science 367, 1362-1366 (2020).

37. Hahn, A. et al. Reconfiguration of functional brain networks and metabolic cost converge during task performance. eLife 9, e52443 (2020).

38. Weiss, A., Chambon, V., Lee, J. K., Drugowitsch, J. \& Wyart, V. Interacting with volatile environments stabilizes hidden-state inference and its brain signatures. http://biorxiv.org/lookup/doi/10.1101/755223 (2019) doi:10.1101/755223.

39. O’Doherty, J. Dissociable Roles of Ventral and Dorsal Striatum in Instrumental Conditioning. Science 304, 452-454 (2004).

40. Garrison, J., Erdeniz, B. \& Done, J. Prediction error in reinforcement learning: A meta-analysis of neuroimaging studies. Neurosci. Biobehav. Rev. 37, 1297-1310 (2013).

41. Grogan, J. P., Sandhu, T. R., Hu, M. T. \& Manohar, S. G. Dopamine promotes instrumental motivation, but reduces reward-related vigour. eLife 9 , e58321 (2020).

42. Gilboa, A. \& Marlatte, H. Neurobiology of Schemas and Schema-Mediated Memory. Trends Cogn. Sci. 21, 618-631 (2017).

43. Klein-Flügge, M. C., Wittmann, M. K., Shpektor, A., Jensen, D. E. A. \& Rushworth, M. F. S. Multiple associative structures created by reinforcement and incidental statistical learning mechanisms. Nat. Commun. 10, 4835 (2019).

44. Jurado-Parras, M. T., Gruart, A. \& Delgado-Garcia, J. M. Observational learning in mice can be prevented by medial prefrontal cortex stimulation and enhanced by nucleus accumbens stimulation. Learn. Mem. 19, 99-106 (2012).

45. Kumaran, D., Warren, D. E. \& Tranel, D. Damage to the Ventromedial Prefrontal Cortex Impairs Learning from Observed Outcomes. Cereb. Cortex 25, 4504-4518 (2015).

46. O’Callaghan, C., Vaghi, M. M., Brummerloh, B., Cardinal, R. N. \& Robbins, T. W. Impaired awareness of action-outcome contingency and causality during healthy ageing and following ventromedial prefrontal cortex lesions. Neuropsychologia 128, 282-289 (2019).

47. Wanke, N. \& Schwabe, L. Subjective Uncontrollability over Aversive Events Reduces Working Memory Performance and Related Large-Scale Network Interactions. Cereb. Cortex N. Y. N 1991 (2019) doi:10.1093/cercor/bhz298.

48. Albares, M. et al. The dorsal medial frontal cortex mediates automatic motor inhibition in uncertain contexts: Evidence from combined fMRI and EEG studies. Hum. Brain Mapp. 35, 5517-5531 (2014).

49. Shenhav, A., Cohen, J. D. \& Botvinick, M. M. Dorsal anterior cingulate cortex and the value of control. Nat. Neurosci. 19, 1286-1291 (2016).

50. Vesuna, S. et al. Deep posteromedial cortical rhythm in dissociation. Nature 586, 87-94 (2020).

51. Li, Y. S., Nassar, M. R., Kable, J. W. \& Gold, J. I. Individual Neurons in the Cingulate Cortex Encode Action Monitoring, Not Selection, during Adaptive Decision-Making. J. Neurosci. 39, 
$6668-6683$ (2019).

52. Leech, R. \& Sharp, D. J. The role of the posterior cingulate cortex in cognition and disease. Brain 137, 12-32 (2014).

53. Wise, T. et al. Instability of default mode network connectivity in major depression: a twosample confirmation study. Transl. Psychiatry 7, e1105 (2017).

54. Leibfried, F., Pascual-Diaz, S. \& Grau-Moya, J. A Unified Bellman Optimality Principle Combining Reward Maximization and Empowerment. ArXiv190712392 Cs Stat (2020).

55. Mohamed, S. \& Jimenez Rezende, D. Variational Information Maximisation for Intrinsically Motivated Reinforcement Learning. Adv. Neural Inf. Process. Syst. 28, 2125-2133 (2015).

56. Dickerson, S. S. \& Kemeny, M. E. Acute stressors and cortisol responses: a theoretical integration and synthesis of laboratory research. Psychol. Bull. 130, 355-391 (2004).

57. Daunizeau, J., Adam, V. \& Rigoux, L. VBA: A Probabilistic Treatment of Nonlinear Models for Neurobiological and Behavioural Data. PLoS Comput. Biol. 10, e1003441 (2014).

58. Hebart, M. N., Görgen, K. \& Haynes, J.-D. The Decoding Toolbox (TDT): a versatile software package for multivariate analyses of functional imaging data. Front. Neuroinformatics 8, (2015). 


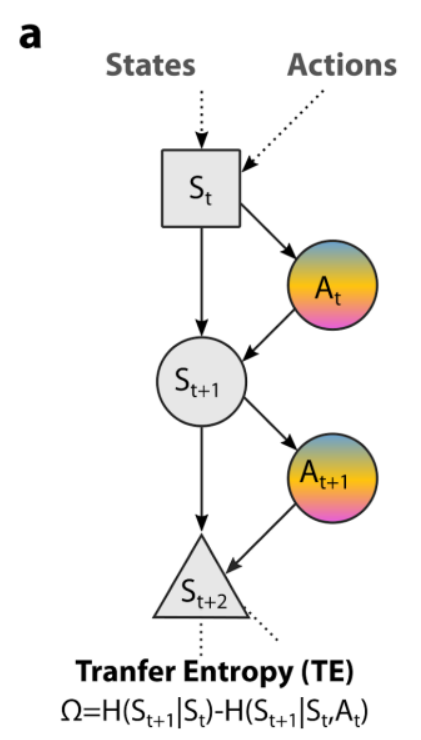

\section{b}

C

Uncontrollable rules: states matter
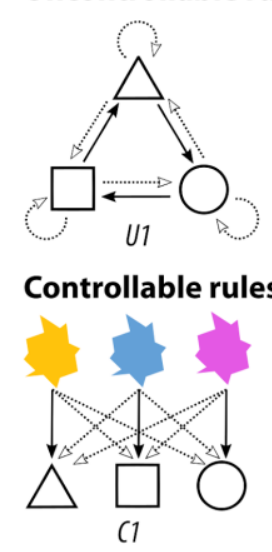

transitions: $\quad \cdots \cdots \cdots$ rare $\longrightarrow$ frequent

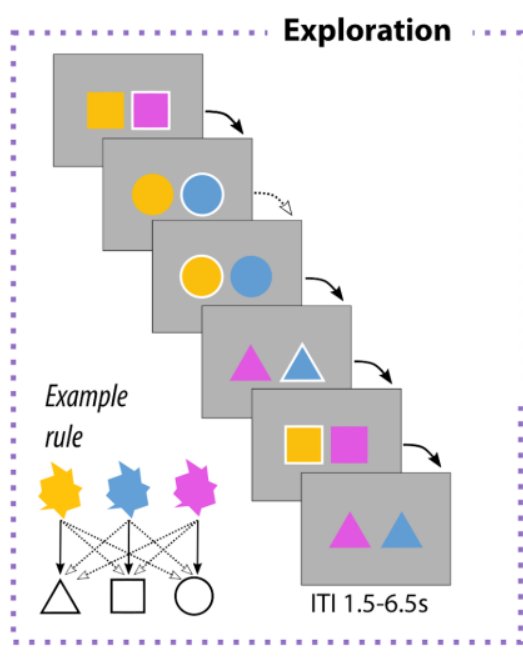

d

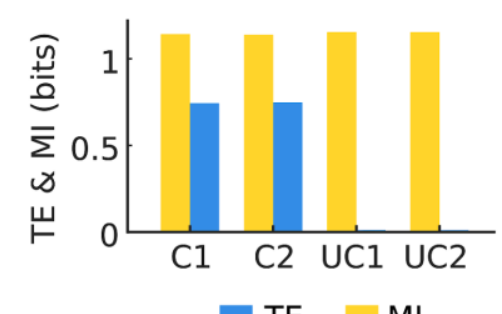

e

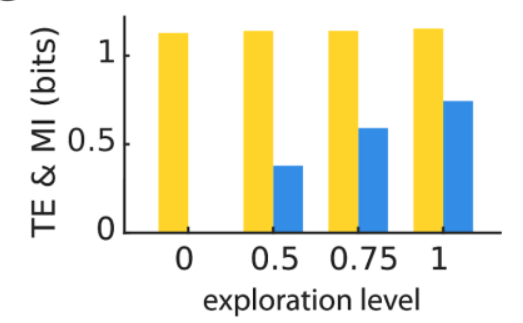

Prediction

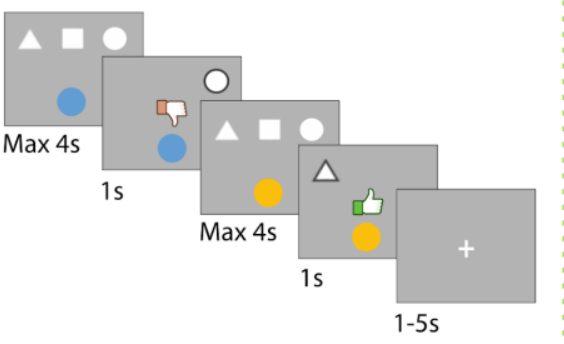

Temporal structure

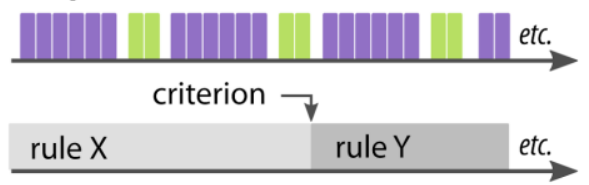

(i)

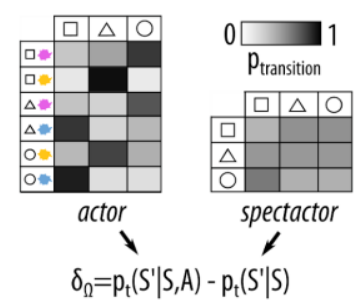

(ii)

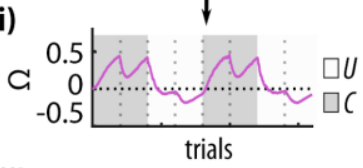

(iii)

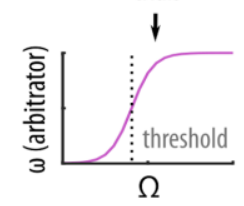

Figure 1. Theoretical framework and experimental protocol. a, Controllability can be inferred from transfer entropy, an information-theoretic measure quantifying to which extent a time series causally influences another one. $\mathbf{b}$, Time course of a novel explore-and-predict task divided in short mini-blocks. Each miniblock consists of a series of exploratory trials (violet) followed by two counterfactual prediction trials (green) used to assess learning and subjective controllability. c, Representation of the 2 uncontrollable rules (U1, U2) and the 2 controllable $(\mathrm{C} 1, \mathrm{C} 2)$ rules, which alternate covertly to govern the evolution of the environment. d, Simulations showing the dissociation of controllability, as indexed by TE, and predictability, as indexed by the mutual information (MI) shared between successive state-action pairs (random exploration policy). e, Simulations under a controllable rule showing that TE requires exploration to be used as a proxy 
for controllability. Indeed, in the absence of exploration, the conditional entropies $H\left(S^{\prime} \mid S\right)$ and $\mathrm{H}\left(\mathrm{S}^{\prime} \mid \mathrm{S}, \mathrm{A}\right)$ are identical (see also Supplementary Note and Supplementary Fig. 1C). f, Synthetic overview of the algorithm able to derive an online approximation of TE (termed $\Omega$ ) by comparing on each trial the transition probabilities of an actor (SAS') and a spectator (SS') model of the world. By thresholding $\Omega$, the algorithm could in turn arbitrate between spectator and actor models when making predictions depending on current controllability estimates $(\omega)$. 

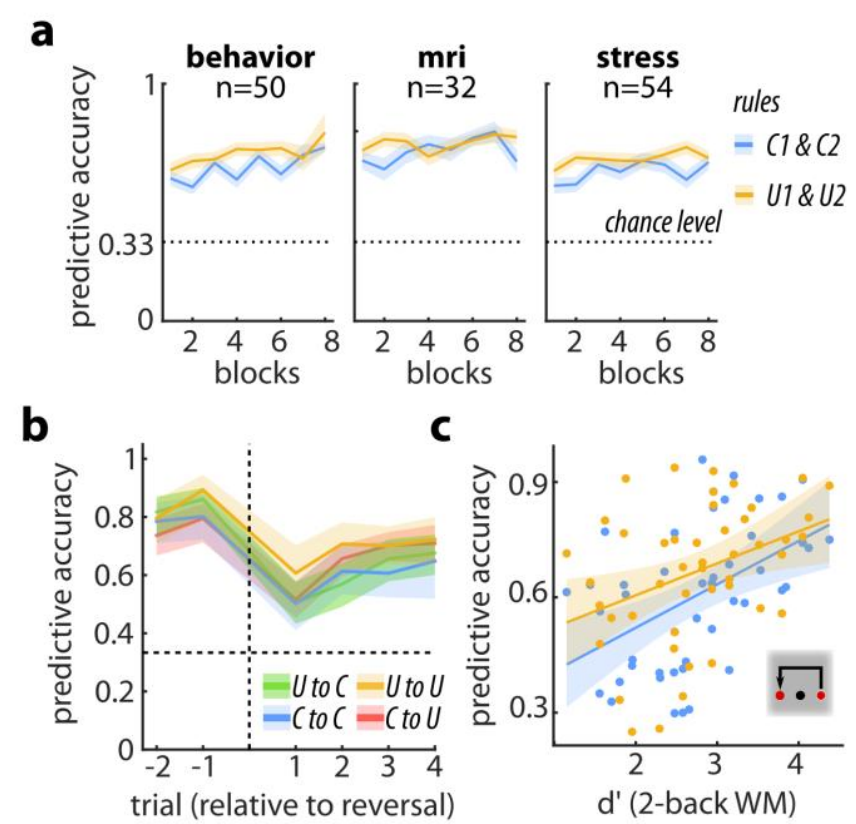

Figure 2. Behavioral performance. a, Accuracy in the prediction trials for each of the 3 experiments, split by condition (see also Supplementary Table 1). b, Fast recovery of predictive accuracy around reversals. c, Positive correlation between working memory capacity indexed by a 2-back task (see Supplementary Methods) and accuracy in the explore-and-predict task for controllable (blue) and uncontrollable (orange) contexts.

Shaded areas represent standard errors of the mean (SEM). 

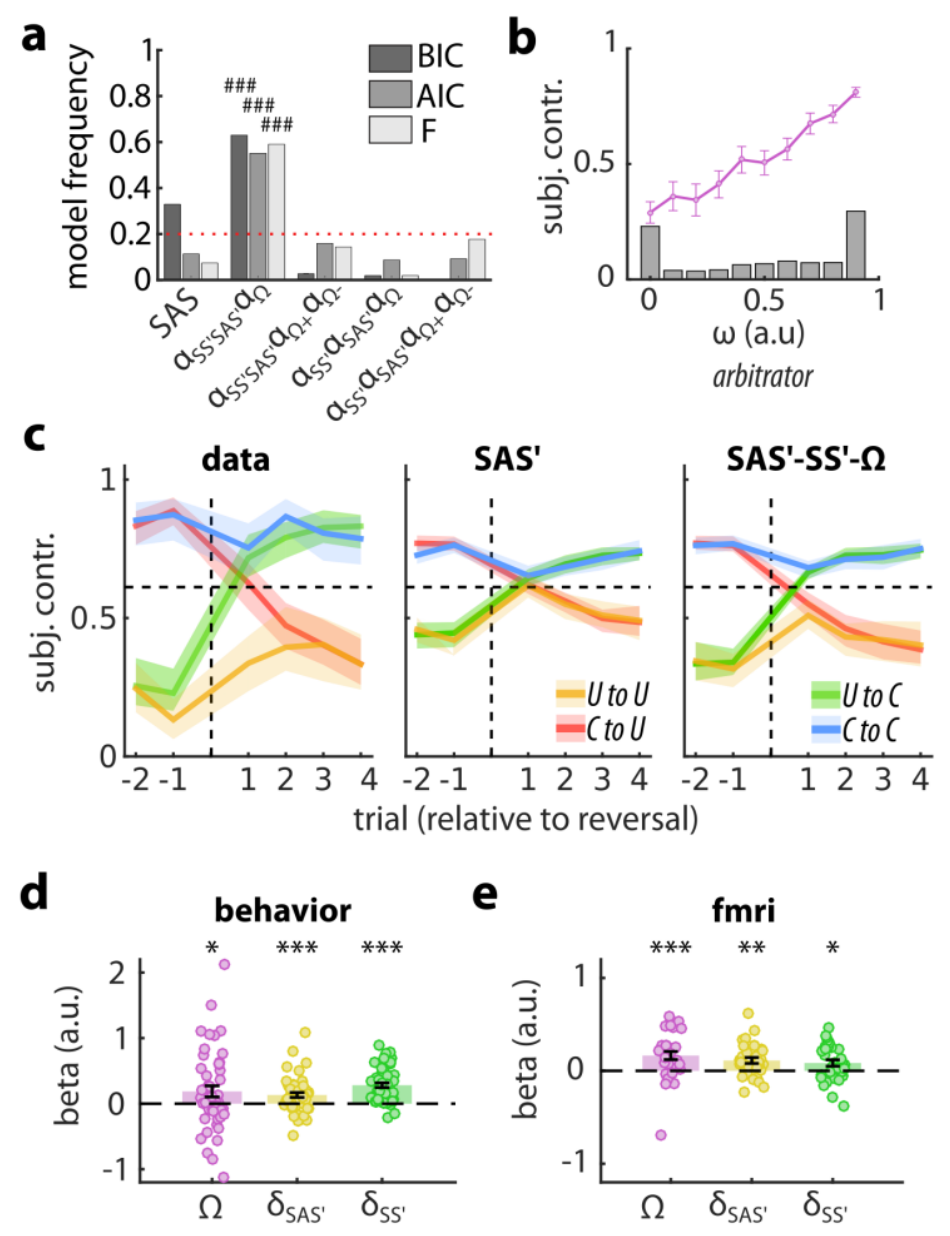

Figure 3. Computational modeling and decision times. a, Bayesian group model comparison pooled over three experiments showed the advantage of the simplest controllability scheme (SS'SAS'- $\Omega$ ) over the actor model (SAS') alone (see Methods and Supplementary Fig. 2a and 3). b, Normalized distribution of the arbitrator variable $\omega$ (grey bar) and its linear relationship with subjective controllability (pink line). Pairs of prediction trials were labelled as "subjectively controllable" when counterfactual predictions differed (e.g. different responses for blue and yellow actions in the circle state). c, The SS'-SAS'- $\Omega$ scheme better captured the dynamics of subjective controllability around reversals. d-e, Coefficients of the logistic regression predicting binarized reaction times in the behavioral and $\mathrm{fMRl}$ experiment using actor and spectator prediction errors ( $\delta$ SAS' and $\delta S S ')$ and $\Omega$.

Error bars and shaded areas represent SEM. ${ }^{*} p<0.05,{ }^{* * *} p<0.005,{ }^{* * *} p<0.001 .{ }^{\# \#} p_{\text {exceedance }}>$ 0.999. BIC: Bayesian Information Criterion. AIC: Akaike Information Criterion. F: Free Energy. 

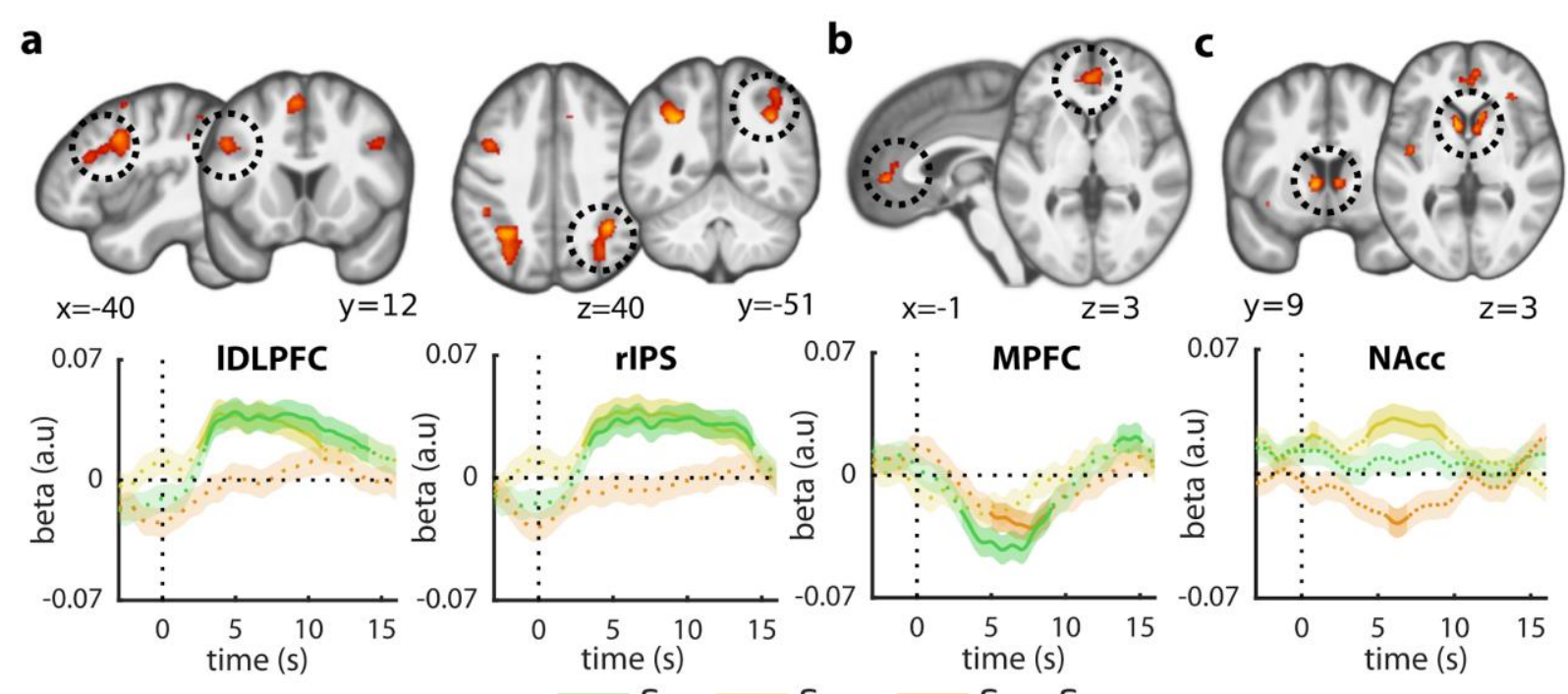

$\delta_{S^{\prime} S^{\prime}} \square \delta_{S^{\prime}}-\delta_{S A S^{\prime}}$

\section{$\delta_{S^{\prime}} \square \delta_{S_{A S}}$}
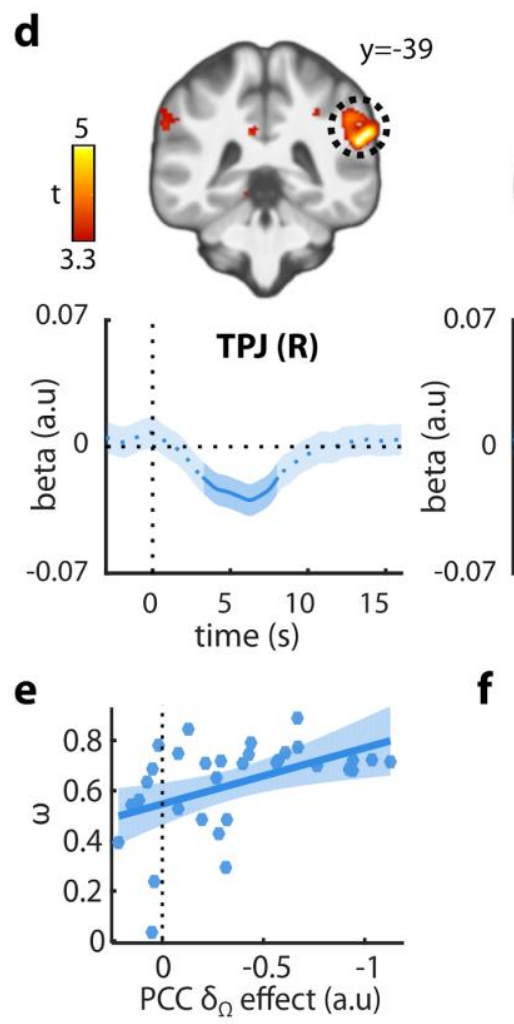

f
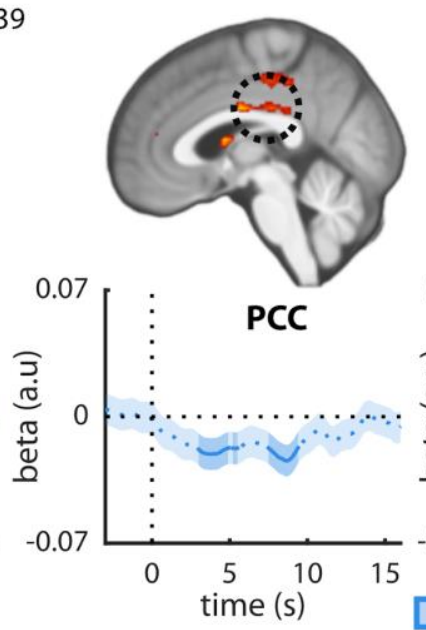

$\mathrm{x}=3$
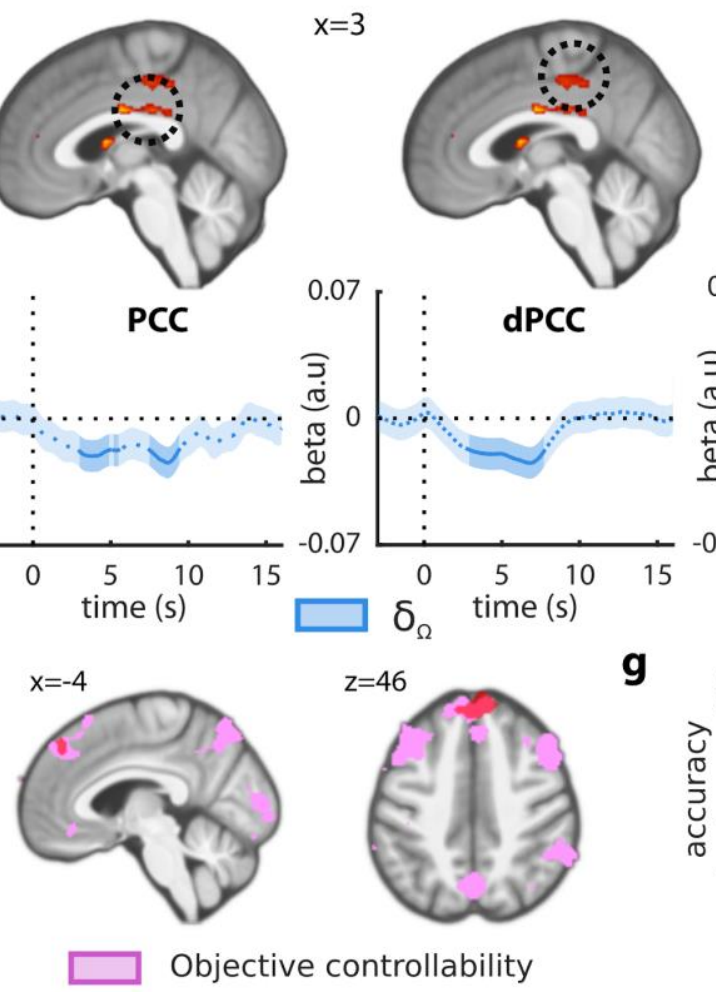
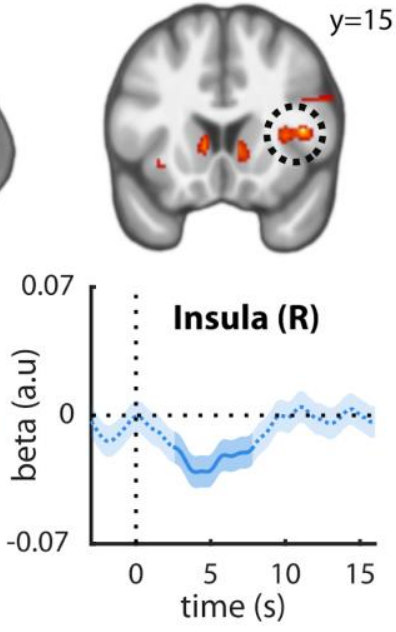

g

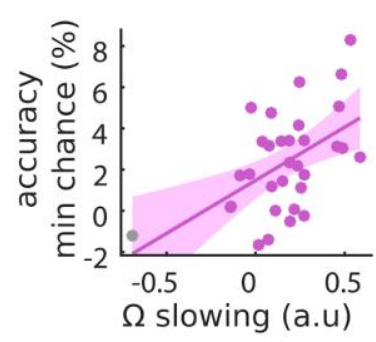

Figure 4. Neuroimaging. a, Brain regions whose activity was higher when the prediction error terms ( $\delta_{\mathrm{SS}}$, and $\delta_{\mathrm{SAS}}$ ) were both above their median value, as compared to when they were both below. b, Paired t-test showing the brain areas whose activity dissociated in response to trials

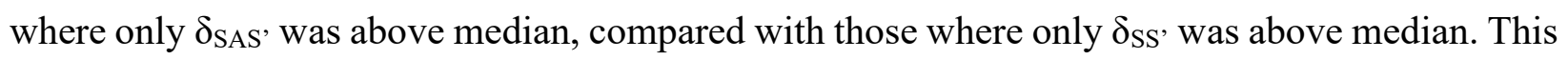
analysis revealed that the mPFC encoded specifically $\delta_{S S}$ ' but not $\delta_{\text {SAS' }}$. c, Parametric analysis of BOLD responses showed that the $\mathrm{mPFC}$ and the nucleus accumbens encoded negatively the 
difference term $\delta_{\mathrm{SS}}$ - $\delta_{\mathrm{SAS}}$, used to update controllability. Contrasting with the mPFC pattern, $\delta_{\mathrm{SAS}}$, was encoded positively In the nucleus accumbens whereas $\delta_{\text {SS' }}$ was not. d, Brain regions encoding signed the second-order prediction errors $\delta_{\Omega}$. All areas surviving correction for multiple comparison showed a negative effect, implying greater activity when an action was less causal than expected. e, The degree to which PCC encoded $\delta_{\Omega}$ predicted the propensity to rely on the actor model across participants. f, Decoding of controllability (rule type) from brain data. A searchlight analysis revealed that the dmPFC, the dlPFC, the right TPJ and the precuneus were sensitive to environmental controllability. g, The sensitivity of the dmPFC to objective controllability predicted to which extent periods of higher controllability led to slower decision times.

The time courses shown below (A-D) were only used for robustness checks and visualization. Statistical inferences were based on whole-brain effects at standard thresholds (voxel-wise: $\mathrm{p}<0.001$, uncorrected; cluster-wise: $\mathrm{p}<0.05^{\mathrm{FWE}}$ ). Shaded areas represent SEM. 
a

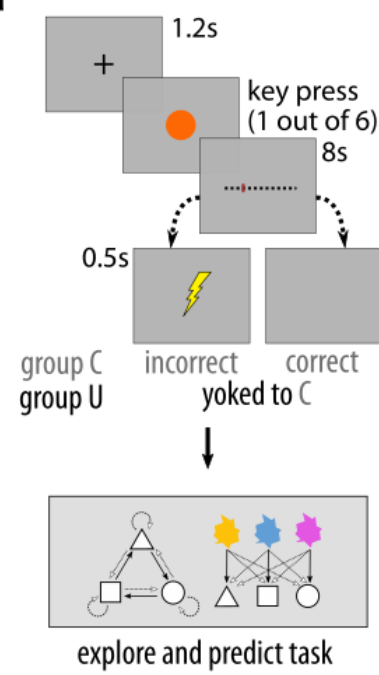

b

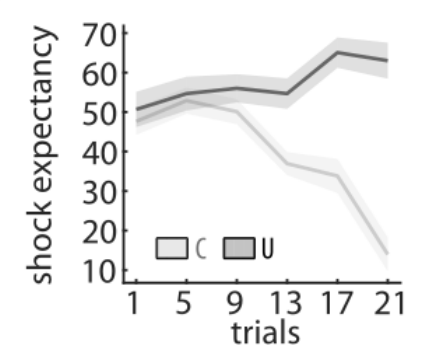

C

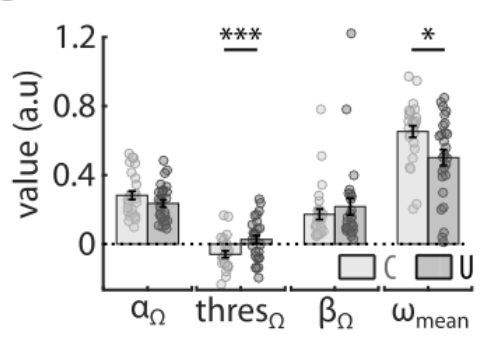

d

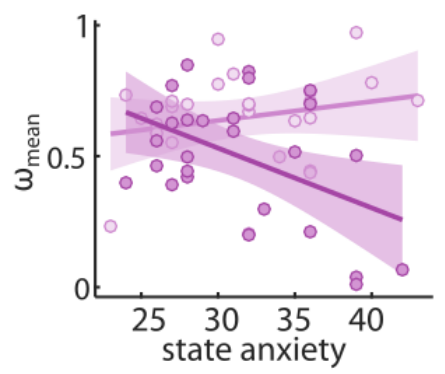

e

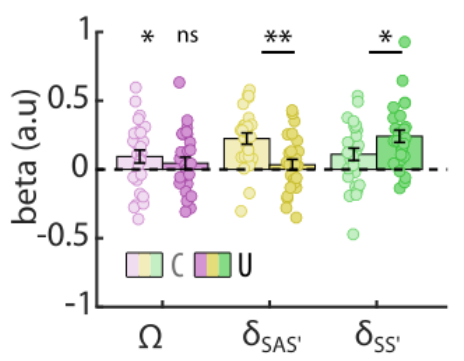

Figure 5. Stress experiment. a, Induction of controllable and yoked uncontrollable stress followed by the explore-and-predict task. b, Temporal evolution shock expectancy during the induction phase, split by condition. c, Impact of induction type on best-fitting parameters related to controllability monitoring as well as on the mean value taken by the arbitrator $\omega$ in the exploreand-predict task. The threshold parameter thres $\Omega$ which determined above which value $\Omega$ was treated as evidence for a controllable environment increased significantly following uncontrollable stressors and the average value of the arbitrator was reduced, hence leading to increased reliance on the spectator model when making predictions $\left(\beta_{\Omega}\right.$ and $\alpha_{\Omega}$ were unaffected, $\beta_{\Omega}$ was divided by 100 for display purposes). d, State anxiety moderated the effect of induction type on the arbitrator variable $(\omega)$ reflecting controllability estimation. Higher state anxiety was associated with greater reliance on the spectator model after exposure to uncontrollable stressors. e, The impact of induction type on the slowing of decision times induced by $\delta_{\mathrm{SS}}$ ', $\delta_{\mathrm{SAS}}$, and $\Omega$ was consistent with increased reliance on the spectator model. Indeed, the effect of $\Omega$ and $\delta_{\mathrm{SAS}}$ ' vanished whereas the effect of $\delta_{\text {ss }}$ increased in the uncontrollable conditions.

All error bars and shaded areas represent SEM. ${ }^{*} \mathrm{p}<0.05,{ }^{* *} \mathrm{p}<0.01$. 